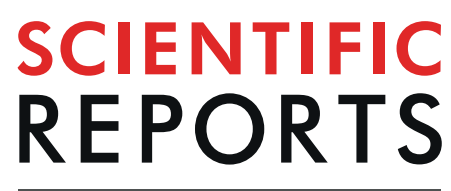

natureresearch

\title{
Complete Protection in Macaques Conferred by Purified Inactivated Zika Vaccine: Defining a Correlate of Protection
}

\author{
GingerYoung $^{1 *}$, Kelly J. Bohning ${ }^{1}$, Melissa Zahralban-Steele ${ }^{1}$, Greg Hather ${ }^{2}$, \\ Sambasivarao Tadepalli ${ }^{3}$, Kristen Mickey ${ }^{4}$, C. Steven Godin ${ }^{5}$, Srisowmya Sanisetty ${ }^{1}$, \\ Stephanie Sonnberg ${ }^{1}$, Hetal K. Patel ${ }^{1}$ \& Hansi J. Dean ${ }^{1}$
}

A critical global health need exists for a Zika vaccine capable of mitigating the effects of future Zika epidemics. In this study we evaluated the antibody responses and efficacy of an aluminum hydroxide adjuvanted purified inactivated Zika vaccine (PIZV) against challenge with Zika virus (ZIKV) strain PRVABC59. Indian rhesus macaques received two doses of PIZV at varying concentrations ranging from $0.016 \mu \mathrm{g}-10 \mu \mathrm{g}$ and were subsequently challenged with ZIKV six weeks or one year following the second immunization. PIZV induced a dose-dependent immune response that was boosted by a second immunization. Complete protection against ZIKV infection was achieved with the higher PIZV doses of $0.4 \mu \mathrm{g}, 2 \mu \mathrm{g}$, and $10 \mu \mathrm{g}$ at 6 weeks and with $10 \mathrm{ug}$ PIZV at 1 year following vaccination. Partial protection was achieved with the lower PIZV doses of $0.016 \mu \mathrm{g}$ and $0.08 \mu \mathrm{g}$. Based on these data, a neutralizing antibody response above $3.02 \log _{10} \mathrm{EC} 50$ was determined as a correlate of protection in macaques. PIZV elicited a dose-dependent neutralizing antibody response which is protective for at least 1 year following vaccination.

In 2015 and 2016, large outbreaks of Zika virus (ZIKV) occurred in the Americas. These outbreaks were associated with clusters of congenital microencephaly and other severe neurological sequelae in infections in approximately 1 of 7 infants born to pregnant women with laboratory confirmed Zika in the US and US territories ${ }^{1}$. Incidence of ZIKV infections subsequently declined in most of the Americas throughout 2017 and $2018^{2}$. With the sporadic nature of ZIKV outbreaks and a very low incidence of symptomatic disease in both endemic and non-endemic areas, conducting phase 3 clinical efficacy trials is not feasible. Still, the risk of re-emergence and the severe consequences of infection in pregnant women demonstrate that the need for an effective Zika vaccine remains. In such circumstances, alternative regulatory strategies such as Animal Rule approval or Accelerated Approval pathway may be relevant for licensure ${ }^{3}$.

Non-human primate studies have contributed to the development of ZIKV vaccines by demonstrating protective efficacy and identifying biomarkers of protection against ZIKV. Results to date have supported neutralizing antibodies as an immune marker that is reasonably likely to predict clinical benefit of several ZIKV vaccines ${ }^{4,5}$. Indian rhesus macaques (Macaca mulatta) are susceptible to ZIKV infection and have been used extensively as a model to study efficacy of ZIKV vaccines and pathogenesis of multiple ZIKV isolates ${ }^{6-10}$. ZIKV infection can be performed by subcutaneous injection, which mimics infection via mosquito bite and causes consistent viremia $^{11-15}$. The kinetics of ZIKV infection are similar in rhesus macaques and humans where serum or plasma viremia typically peaks within the first six days of infection and resolves within 10-14 days ${ }^{10,13,14}$.

The purified inactivated Zika vaccine (PIZV) has previously been evaluated in mouse models and was immunogenic in AG129 and CD1 mice and protected AG129 mice against lethal ZIKV challenge ${ }^{16}$. In those studies, Baldwin et al. demonstrated that neutralizing antibodies correlate with protection in AG129 mice. PIZV is currently being evaluated for safety and immunogenicity in phase 1 trials (ClinicalTrials.gov NCT03343626).

\footnotetext{
${ }^{1}$ Takeda Vaccines, Inc., Cambridge, MA, USA. ${ }^{2}$ Takeda Pharmaceuticals, Inc., Cambridge, MA, USA. ${ }^{3}$ Charles River Laboratories, Mattawan, MI, USA. ${ }^{4}$ Viracor Eurofins Lee's Summit, Missouri, MO, USA. ${ }^{5}$ Inotiv, Gaithersburg, MD, USA. *email: ginger.young@takeda.com
} 


\begin{tabular}{|l|l|l|l|l|l|l|l|l|}
\hline \multirow{2}{*}{$\begin{array}{l}\text { Challenge } \\
\text { Dose }(\mathbf{f f u})\end{array}$} & \multicolumn{6}{l}{ Zika vRNA $\left(\log _{10}\right.$ copies/mL) } \\
\cline { 2 - 9 } & Day 1 & Day 2 & Day 3 & Day 4 & Day 5 & Day 6 & Day 7 & Day 8 \\
\hline \multirow{3}{*}{$10^{4}$} & UD & $<$ LLOQ & 4.5 & 4.9 & 4.8 & 4.6 & 3.5 & 2.9 \\
\cline { 2 - 9 } & UD & UD & 4.6 & 4.9 & 5.6 & 5.4 & UD & $<$ LLOQ \\
\hline \multirow{2}{*}{$10^{5}$} & UD & 3.3 & 4.6 & 4.8 & 4.3 & 3.8 & UD & UD \\
\cline { 2 - 9 } & UD & 3.8 & 4.3 & 5.0 & 5.0 & 4.0 & UD & UD \\
\hline
\end{tabular}

Table 1. Serum vRNA per challenge dose and day post-challenge. Zika vRNA $\left(\log _{10} \operatorname{copies} / \mathrm{mL}\right)$ per day post-challenge for each individual macaque. Zika vRNA for individual macaques was not quantified in the $10^{4} \mathrm{ffu}$ dose after day 8 or the $10^{5} \mathrm{ffu}$ challenge dose group after day 6 post-challenge. UD =Undetected. $<$ LLOQ $=$ detected vRNA was below the assay lower limit of quantitation.

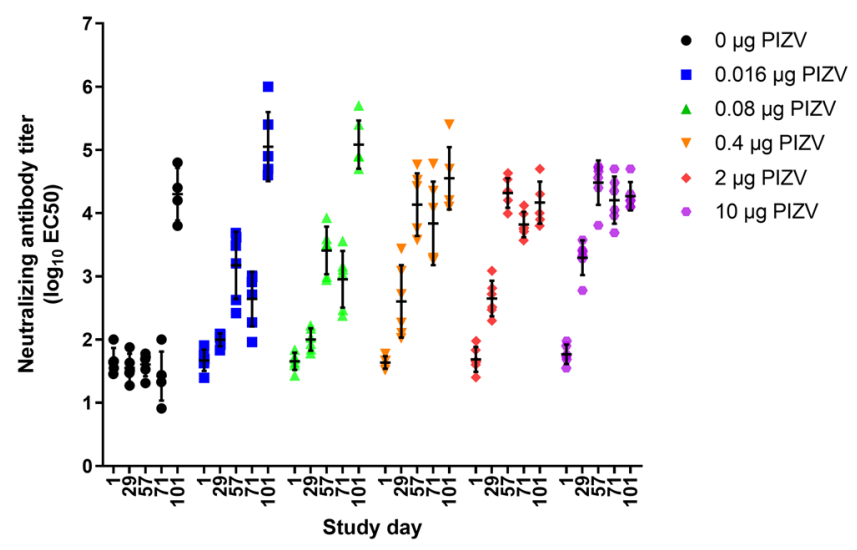

Figure 1. Neutralizing antibody titers following PIZV vaccination and ZIKV challenge. Rhesus macaques were vaccinated on days 1 and 29 and challenged on day 71. Individual Zika RVP titers for each vaccine group on study day 1 (prior to vaccination), day 29 (28 days post-first dose), day 57 (28 days post-second dose), day 71 (prior to ZIKV challenge), and day 101 (30 days post-ZIKV challenge). Mean and standard deviation are shown for all groups and time points.

To further evaluate immunogenicity and efficacy of PIZV, we conducted three ZIKV challenge studies in rhesus macaques. In the first study, we established a dose of PRVABC59 challenge virus. In the second study, we determined the immunogenicity and efficacy of a wide range of PIZV dose levels at 42 days after two PIZV vaccinations, to establish a potential antibody correlate of protection. In the third study, we assessed the persistence of immunity and efficacy 1 year following administration of the second PIZV dose, to evaluate neutralizing antibody kinetics and long-term protection.

\section{Results}

Challenge dose selection. We conducted a challenge study to select a ZIKV challenge dose that appropriately mimics human infection in Indian rhesus macaques. Macaques were challenged via subcutaneous injection with $0.5 \mathrm{~mL}$ containing either $10^{4}$ focus forming units ( $\left.\mathrm{ffu} ; \mathrm{n}=2\right)$ or $10^{5} \mathrm{ffu}(\mathrm{n}=2)$ ZIKV PRVABC59. Serum was collected daily for ZIKV RNA analysis by real-time quantitative RT-PCR (RT-qPCR), on days 1-11 post-infection (dpi), and every other day from day 13-21 dpi. Zika viral RNA (vRNA) was detected above the assay lower limit of quantitation (LLOQ) between days $3-8$ in the $10^{4} \mathrm{ffu}$ dose group and between days $2-6$ in the $10^{5} \mathrm{ffu}$ challenge dose group (Table 1). Macaques receiving the $10^{4} \mathrm{ffu}$ challenge dose had peak vRNA of 4.9 and $5.6 \log _{10}$ copies/ $\mathrm{mL}$ on days 4 and 5, while macaques in the $10^{5} \mathrm{ffu}$ group had peak vRNA of 4.8 and $5.0 \log _{10} \operatorname{copies} / \mathrm{mL}$, both on day 4. Zika vRNA was not detected above the assay LLOQ in the $10^{4} \mathrm{ffu}$ challenge dose group after day 8 or in the $10^{5} \mathrm{ffu}$ challenge dose group after day 6 . The $10^{4} \mathrm{ffu}$ challenge dose was selected as it resulted in higher peak vRNA and longer duration of vRNA detection than the $10^{5} \mathrm{ffu}$ challenge dose.

PIZV elicits a dose dependent neutralizing antibody response. Rhesus macaques were vaccinated with PIZV on days 1 and 29 and challenged on day 71. Serum neutralizing antibodies were tested using a Zika reporter virus particle (RVP) assay. All macaques were seronegative on day 1 and control macaques remained seronegative prior to ZIKV challenge (Fig. 1). Twenty-eight days following the first vaccine dose (study day 29), little or no seroconversion was observed for the 0.016 and $0.08 \mu \mathrm{g}$ PIZV doses, while $4 / 6$ macaques vaccinated with the $0.4 \mu \mathrm{g}$ PIZV dose seroconverted and 6/6 macaques receiving the 2 and $10 \mu \mathrm{g}$ PIZV dose seroconverted. Immune responses were boosted in all groups after the second dose (all $p$-values $\leq 0.001$ ). Twenty-eight days after the second PIZV dose (study day 57), 100\% of vaccinated macaques seroconverted. The magnitude of neutralizing antibody titers on day 57 was similar after the second dose of $0.4,2$, or $10 \mu \mathrm{g}$, with no statistically significant difference detected among these groups. The titer on day 57 was lower in the $0.016(p=0.003)$ and $0.08 \mu$ groups 


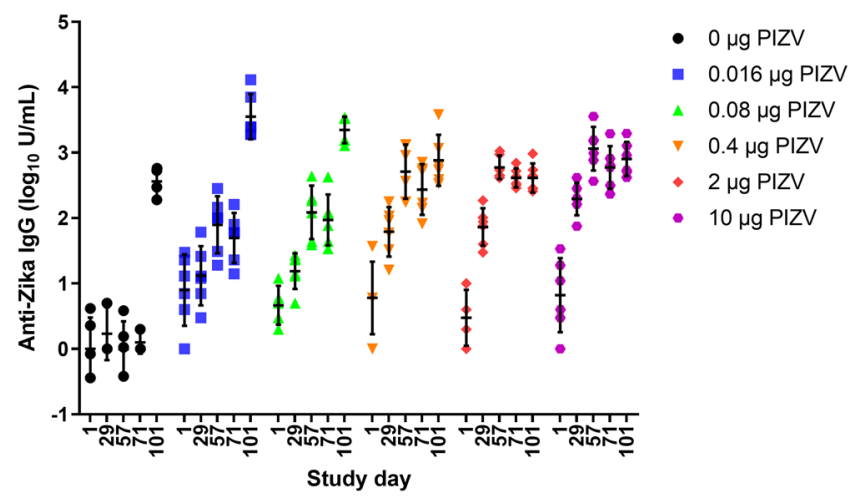

Figure 2. Anti-Zika IgG response following PIZV vaccination. Anti-Zika IgG concentrations for each vaccine group on study day 1 (prior to vaccination), day 29 (28 days post-first dose), day 57 (28 days post-second dose), day 71 (prior to ZIKV challenge), and day 101 (30 days post-ZIKV challenge). Mean and standard deviation are shown for all groups and time points.

$(p=0.037)$ compared to the $0.4 \mu$ g dose group. Titers prior to ZIKV challenge on day 71 were slightly decreased compared to day 57 . Following challenge, neutralizing antibody titers increased significantly in the placebo, 0.016 and $0.08 \mu \mathrm{g}$ groups (all p-values $\leq 0.004$ ), but not in the $0.4,2$, and $10 \mu \mathrm{g}$ groups. Neutralizing antibody levels after two PIZV doses of $0.4,2$, or $10 \mu \mathrm{g}$ were similar to post-challenge neutralizing antibody titers in the placebo group. In conclusion, we observed a dose-dependent neutralizing antibody response to one or two doses of PIZV. After two PIZV doses, the neutralizing antibody levels reached a plateau for vaccine doses above $0.4 \mu \mathrm{g}$, and the level of neutralizing antibody was comparable to infection with ZIKV challenge in the placebo group. The lack of anamnestic antibody responses after challenge in the $0.4,2$, and $10 \mu \mathrm{g}$ PIZV dose groups suggests that infection by ZIKV challenge virus may have been prevented by vaccination.

PIZV elicits dose-dependent anti-Zika IgG responses. In addition to evaluating neutralizing antibodies, we also quantified anti-Zika-specific IgG using a Luminex-based assay. As with neutralizing antibodies, all PIZV doses were immunogenic and no anti-Zika IgG was detected in the control group prior to ZIKV challenge. PIZV elicited dose-dependent anti-Zika IgG responses (Fig. 2) in the vaccinated groups. Anti-Zika IgG responses were significantly boosted after a second PIZV dose for all vaccinated groups (all p-values $\leq 0.001$ ). Following ZIKV challenge, the anti-Zika IgG responses increased significantly in the placebo, 0.016 and $0.08 \mu g$ groups (all p-values $\leq 0.035$ ), but not in the $0.4,2$, and $10 \mu \mathrm{g}$ groups. Similar to the neutralizing antibody response, the anti-Zika IgG titers following vaccination with the $0.4,2$, or $10 \mu \mathrm{g}$ doses were similar to post-challenge anti-Zika IgG titers in the placebo group.

PIZV protects against ZIKV challenge. Rhesus macaques were challenged subcutaneously on day 71 with $10^{4} \mathrm{ffu}$ PRVABC59. All macaques receiving the 0.4 , 2, or $10 \mu \mathrm{g}$ PIZV dose were protected against ZIKV challenge, as no Zika vRNA could be quantified in any of the macaques in these groups. Zika vRNA was quantified in 3/6 macaques receiving the $0.08 \mu \mathrm{g}$ dose, $4 / 6$ macaques receiving the $0.016 \mu \mathrm{g}$ dose, and in all macaques in the control group (Fig. 3). The peak post-challenge Zika vRNA level decreased with increasing vaccine dose level. The peak Zika vRNA occurred on day 74 (3 days post-challenge), with a geometric mean level of $5.1 \log _{10}$ copies/ $\mathrm{mL}$ in the control group (range $4.92-5.64 \log _{10}$ copies $/ \mathrm{mL}$ ), $4.1 \log _{10}$ copies $/ \mathrm{mL}$ in the $0.016 \mu$ g dose group (range 4.10-5.00 $\log _{10}$ copies $/ \mathrm{mL}$ ) and $3.2 \log _{10}$ copies $/ \mathrm{mL}$ in the $0.08 \mu \mathrm{g}$ dose group (range $2.98-3.33 \log _{10}$ copies $/ \mathrm{mL}$ ). No clinical signs to the vaccine or challenge virus were seen throughout the studies.

Correlate of protection. PIZV elicited a dose dependent neutralizing antibody immune response and an anti-Zika IgG response which correlated with a reduction in ZIKV vRNA post-challenge (Table 2). An immune correlate analysis was subsequently performed to correlate Zika vRNA with neutralizing antibody titers (Fig. 4) and anti-Zika IgG (Fig. 5), which demonstrated that an increase in both neutralization antibody and anti-Zika IgG titers correlates with a decrease in vRNA concentration. Correlation with protection was observed with both neutralizing antibodies $\left(3.02 \log _{10}\right.$ EC50) and anti-Zika $\operatorname{IgG}\left(2.21 \log _{10} \mathrm{U} / \mathrm{mL}\right)$. We chose the functional assay, neutralizing antibodies, to establish a correlate of protection in macaques as a neutralizing antibody titer of $>3.02$ $\log _{10}$ EC50, which conferred protection against ZIKV serum viremia in Indian rhesus macaques. Due to overlap among the distributions of neutralizing antibody titers between the protected and infected animals, titers in some protected animals were below the correlate of protection.

Immunogenicity and efficacy 1 year post-vaccination. To determine the kinetics of neutralizing antibodies over a year long period, a separate set of four male Indian rhesus macaques were vaccinated with $10 \mu \mathrm{g}$ PIZV on study days 1 and 29 and challenged with ZIKV PRVABC59 on study day 371. Zika neutralizing antibody levels were measured every 28 days through day 365 (except for days 197, 309, and 337), as well as on day 401 (30 days post-ZIKV challenge). All macaques seroconverted following the first vaccine dose, with a significant boost in antibody titers following the second dose ( $p<0.001$; Fig. $6 a)$. Neutralizing antibody titers peaked on day 57,28 days following the second immunization, declined from day 57 to day 85 , and then remained stable from day 85 
PIZV $0 \mu g$

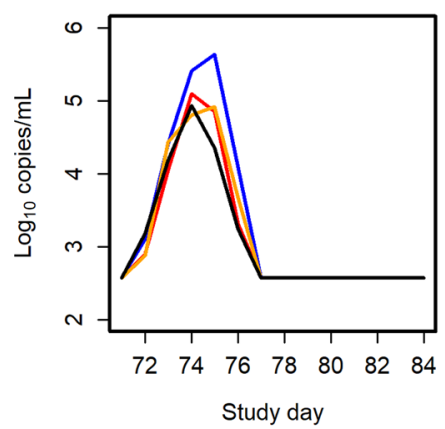

PIZV $0.4 \mu g$

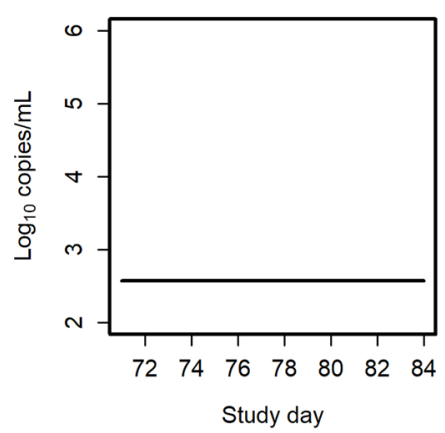

PIZV $0.016 \mu g$

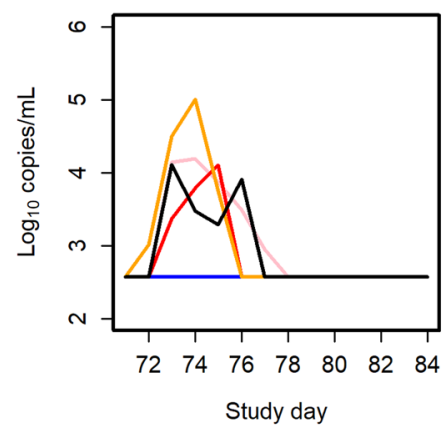

PIZV $2 \mu g$

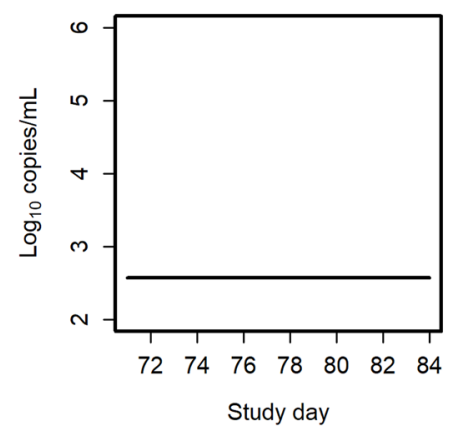

PIZV $0.08 \mu g$

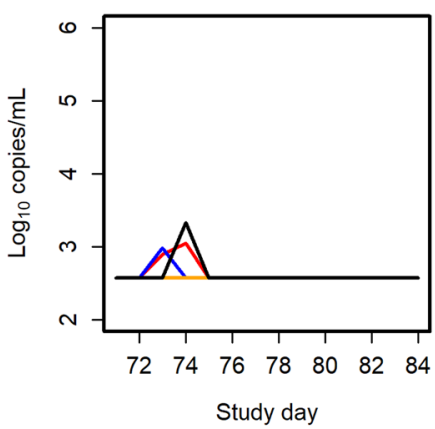

PIZV $10 \mu g$

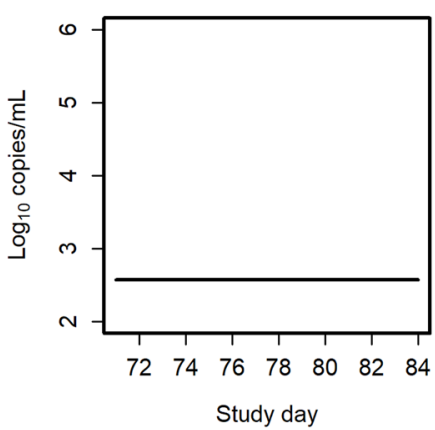

Figure 3. Post-challenge Zika vRNA per group and study day. Zika vRNA ( $\log _{10}$ copies $\left./ \mathrm{mL}\right)$ post-ZIKV challenge. Each line represents an individual macaque. Zika vRNA levels below the LLOQ are plotted as half of the LLOQ. Within each plot, each color represents an individual macaque.

\begin{tabular}{|l|l|l|l|}
\hline $\begin{array}{l}\text { PIZV dose } \\
(\boldsymbol{\mu g})\end{array}$ & $\begin{array}{l}\text { Mean neutralizing antibody } \\
\text { titer }\left(\log _{10} \text { EC50), day } 71\right.\end{array}$ & $\begin{array}{l}\text { Range of peak Zika vRNA } \\
\text { detection }\left(\log _{10} \text { copies/mL) }\right.\end{array}$ & $\begin{array}{l}\text { Percent macaques protected } \\
\text { from ZIKV challenge }\end{array}$ \\
\hline 0 & 1.33 & $4.92-5.64$ & 0 \\
\hline 0.016 & 2.63 & $4.10-5.00$ & 33 \\
\hline 0.08 & 2.94 & $2.98-3.33$ & 50 \\
\hline 0.4 & 3.81 & $<$ LLOQ & 100 \\
\hline 2 & 3.81 & $<$ LLOQ & 100 \\
\hline 10 & 4.19 & $<$ LLOQ & 100 \\
\hline
\end{tabular}

Table 2. Neutralizing antibody titers on day of ZIKV challenge, Zika vRNA levels post-challenge. Summary of mean neutralizing antibody titers $\left(\log _{10}\right.$ EC50), range of peak vRNA post-ZIKV challenge $\left(\log _{10} \operatorname{copies} / \mathrm{mL}\right)$, and percent of macaques with quantifiable Zika vRNA post-ZIKV challenge. All reported and analyzed data is above the RT-qPCR LLOQ. $<$ LLOQ = detected vRNA was below the assay lower limit of quantitation.

to day 401. Anti-Zika IgG levels also peaked on day 57 and subsequently declined through study day 365 (Fig. 6b). IgG titers increased following ZIKV challenge.

No Zika vRNA was detected in serum from any of the macaques following ZIKV challenge 1 year following the second dose. In addition, no statistically significant increase in neutralizing antibodies was observed post-ZIKV challenge on day 401 ( $\log _{10}$ EC50 range of 3.72-4.31 on day 365 compared to $\log _{10}$ EC50 range of $4.00-4.49$ on day 401). The combined results suggest that two doses of PIZV prevented ZIKV infection 1 year post-vaccination. In conclusion, two $10 \mu \mathrm{g}$ PIZV vaccinations elicits persistent neutralizing antibodies and long-term protection in rhesus macaques.

\section{Discussion}

We demonstrated that PIZV elicits a dose-dependent response of both Zika neutralizing and anti-Zika IgG antibodies. PIZV elicited neutralizing antibodies that persisted for at least 1 year and protected against ZIKV challenge. Vaccinating with a broad range of PIZV dose levels enabled us to correlate both neutralizing and anti-Zika IgG antibody titers to protection against ZIKV infection. We determined a neutralizing antibody correlate of protection of $3.02 \log _{10}$ EC50, which we define as the maximum EC50 among the unprotected animals in the study. We chose the neutralizing antibody assay to establish a correlate of protection as the literature supports using functional neutralizing antibodies as a correlate of protection for Zika and other flaviviruses. 

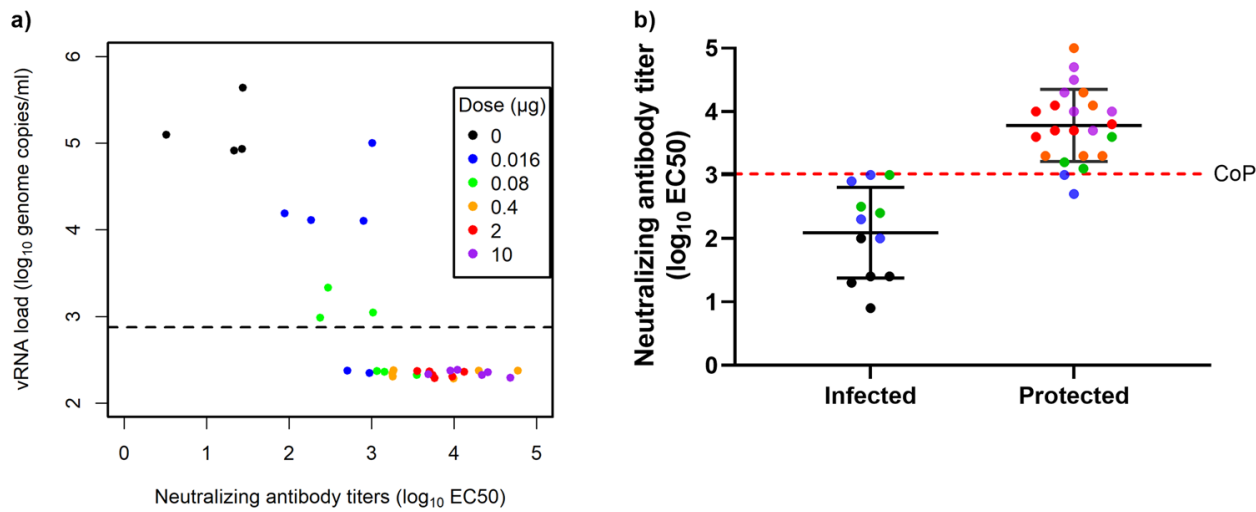

Figure 4. Neutralizing antibody titers negatively correlate with Zika vRNA peak value post-challenge. (a) Each point represents an individual macaque and respective neutralizing antibody titer $\left(\log _{10}\right.$ EC50) on day of ZIKV challenge (day 71$)$ and peak ZIKV vRNA concentration $\left(\log _{10}\right.$ copies $\left./ \mathrm{mL}\right)$ post-ZIKV challenge. For each macaque, the maximum Zika vRNA concentration observed over 10 days was plotted for analysis. Zika vRNA levels below the LLOQ are plotted as half of the LLOQ. The LLOQ is shown as a dashed line. To prevent overlaps among the points below the LLOQ, a small amount of variation has been added in the vertical direction. (b) To determine the correlate of protection, the neutralizing antibody titers $\left(\log _{10}\right.$ EC50) of infected (positive for vRNA at any given timepoint) and protected (negative for vRNA) macaques were plotted. The correlate of protection was defined as the maximum neutralizing antibody titer across all unprotected macaques in this study. Based on this definition, the correlate of protection was established as $>3.02 \log _{10} \mathrm{EC} 50$. CoP $=$ Correlate of protection.
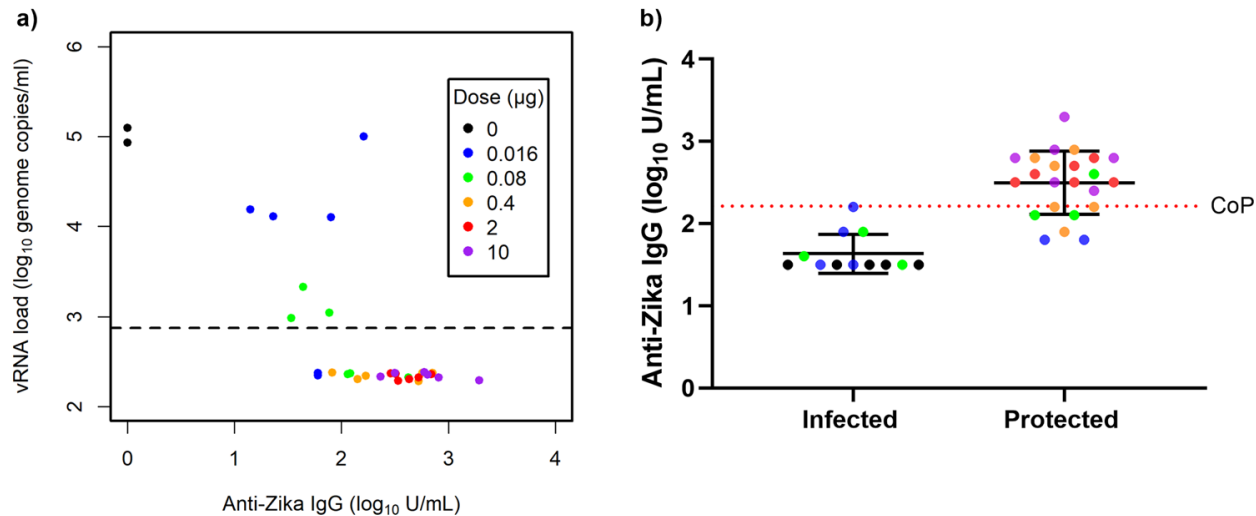

Figure 5. Anti-Zika IgG antibody titers negatively correlate with Zika vRNA post-challenge. (a) Each point represents an individual macaque. This plot demonstrates the relationship between Day 71 anti-Zika IgG and the peak vRNA concentration following ZIKV challenge. Zika vRNA values below the LLOQ were replaced by half of the LLOQ. The LLOQ is shown as a dashed line. To prevent overlaps in the plot, these data points have been jittered in the vertical direction. (b) To determine the correlate of protection for anti-Zika IgG, the IgG antibody titers $\left(\log _{10} \mathrm{U} / \mathrm{mL}\right.$ ) of infected (positive for vRNA at any given timepoint) and protected (negative for vRNA) macaques were plotted. Mean and standard deviation are shown for all groups and time points. The correlate of protection for anti-Zika IgG is $2.21 \log _{10} \mathrm{U} / \mathrm{mL}$ and was defined as the maximum anti-Zika IgG across all unprotected macaques in this study. $\mathrm{CoP}=$ Correlate of protection.

Neutralizing antibodies directed against the envelope (E) protein have been identified as correlates of protection for vaccines Japanese encephalitis virus (JEV), yellow fever virus, and tickborne encephalitis viruses ${ }^{4}$. Other laboratories using different vaccine platforms (DNA, RNA, inactivated virus, protein subunit, adenovirus-vectored, VLP) have reported induction of ZIKV-specific neutralizing antibodies that conferred protection against live ZIKV challenge in animal models ${ }^{6-8,16-22}$. These data support ZIKV E-specific neutralizing antibodies as a mechanism of protection against ZIKV infection.

In flavivirus vaccine development, neutralizing antibody titers that can confer protection against viremia have be reported in animal models using several different assay methods: microneutralization (MNT), RVP, and plaque reduction neutralization (PRNT). Studies of other candidate ZIKV vaccines have demonstrated that a neutralizing antibody titer as low as 100, using a MNT assay, or a titer of 1,000 using an RVP assay can confer protection against viremia in animal models ${ }^{6,16,22}$. These results are qualitatively similar to those of licensed vaccines against flaviviruses such as JEV, where a neutralizing antibody titer of $>10$ (as measured by PRNT) is considered 

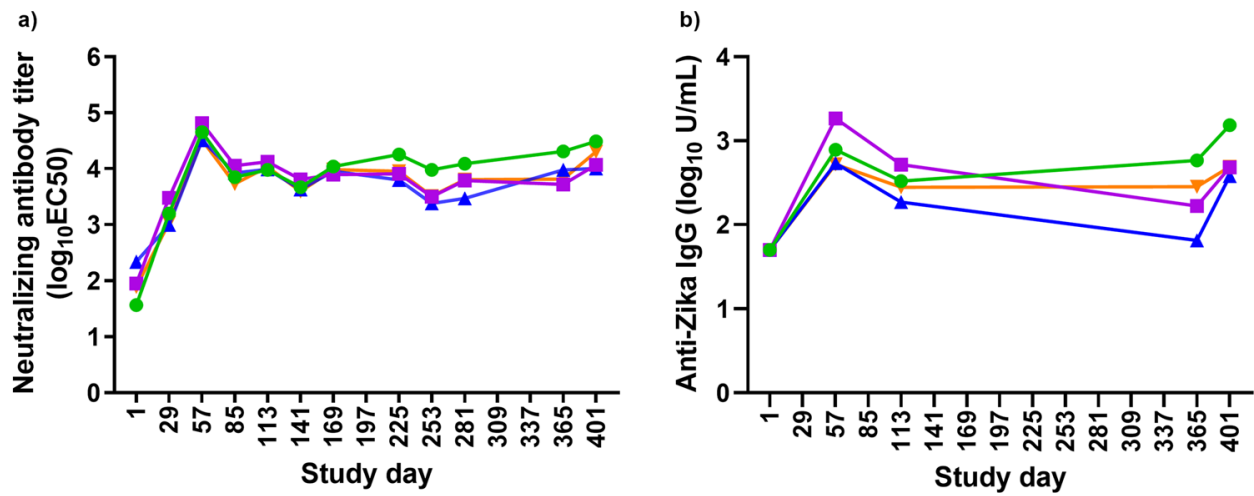

Figure 6. Long-term immunogenicity following PIZV vaccination. Both neutralizing antibody (a) and antiZika IgG titers were determined (b). Macaques were vaccinated on days 1 and 29 and challenged on day 371. Key time points include study day 1 (prior to vaccination), day 29 (28 days post-first dose), day 57 (28 days post-second dose), day 365 (prior to ZIKV challenge), and day 401 (30 days post-ZIKV challenge). Each line represents an individual macaque.

to correlate with protection ${ }^{23-25}$. Further supporting this correlate of protection, it has been reported that passive transfer of Zika neutralizing monoclonal antibodies or antisera from vaccinated humans and animals to mice is sufficient to protect against ZIKV challenge ${ }^{16,17,21,26}$. Adoptive transfer of serum from immunized mice fully protected against viremia, while splenocytes from the same donors provided marginal protection ${ }^{27}$ demonstrating that the mechanism of protection against ZIKV infection is antibody mediated.

While ZIKV exists as three genotypes (West African, East African and Asian), they all behave as a single serotype $^{28,29}$. Our previous studies demonstrated that PIZV is capable of eliciting antibodies that neutralize both African and Asian ZIKV isolates in vitro ${ }^{16}$. Others have shown that the magnitude or duration of viremia in unvaccinated macaques challenged with ZIKV isolates from Brazil or Puerto Rico is similar ${ }^{6}$, and that vaccinated macaques are protected against challenge with heterologous ZIKV strains ${ }^{13,21,30}$. Protection of animal models against heterologous challenge and cross-neutralization capabilities of antisera from multiple vaccine platforms ${ }^{8,17,18,21}$ suggest that data from a single challenge strain may be sufficient to show cross protection against ZIKV strains from other lineages. Several groups have developed rhesus macaque challenge models using Zika strain PRVABC596,7,9,12,31 which is a well-documented and characterized isolate from human serum and is representative of viruses that were circulating in the Americas during the 2015-2016 outbreak ${ }^{16}$. We therefore selected PRVABC59 as the challenge strain for our studies.

In this study, we extended our observation of PIZV efficacy in mice ${ }^{16}$ to demonstrate efficacy in prevention of ZIKV vRNA and to evaluate anamnestic antibody responses after ZIKV challenge in non-human primates. We did not assess presence of vRNA in tissues. Our working hypothesis is that prevention of serum ZIKV vRNA may be a surrogate for prevention of the most serious sequelae of ZIKV infection in humans - fetal infection. By employing a PIZV dose titration study design and a ZIKV RVP assay, we have established a minimum protective vaccine dose of $0.4 \mu \mathrm{g}$ in rhesus macaques and established a neutralizing antibody correlate of protection against ZIKV challenge of $3.02 \log _{10}$ EC50. Finally, we demonstrated that ZIKV neutralizing antibodies persist and are capable of preventing ZIKV infection for at least 1 year post-vaccination. In the event that human phase 3 efficacy studies are not feasible, identifying a correlate of protection in an appropriate non-human primate challenge model may be important to support licensure of a Zika vaccine ${ }^{3,5,32}$. Altogether, these data support neutralizing antibodies as an immune marker that is associated with efficacy in a relevant animal model and that may predict a reasonable likelihood of clinical benefit in humans.

\section{Methods}

Vaccine. PIZV is an aluminum hydroxide adjuvanted whole purified inactivated virus vaccine based on ZIKV strain PRVABC59 which was originally isolated from serum from a human infected in Puerto Rico (GenBank accession number KU501215). PIZV has been previously described and characterized by Baldwin et al. ${ }^{16}$. The same lot of PIZV used in this study was also used in clinical trial ZIK101 (ClinicalTrials.gov NCT03343626).

Rhesus macaque challenge studies. Thirty-five Indian rhesus macaques were separated into 6 groups. Three male and three female macaques per group were vaccinated intramuscularly with either 0.016, 0.08. 0.4, 2 or $10 \mu \mathrm{g}$ PIZV on study days 1 and 29. To achieve statistical significance comparing vaccine doses, six animals/ group were selected to obtain $>80 \%$ power if the true infection rate among controls is $\geq 90 \%$ and the true infection rate among vaccinated animals is $\leq 16 \%$ based on a one-sided Fisher's Exact Test with $5 \%$ Type 1 error rate . $^{7}$ Two male and three female macaques were vaccinated with placebo control (PBS) on study days 1 and 29. One female from the control group was euthanized on the day of ZIKV challenge due to reasons unrelated to the study, resulting in 4 macaques in the control group. Macaques were challenged subcutaneously with $10^{4} \mathrm{ffu} / 0.5 \mathrm{~mL}$ ZIKV PRVABC59 on study day 71 . Serum samples were collected and tested for antibody titers on study days 1 , 29,57 , and 71, and 101, and for ZIKV RNA on study days 71-80, and study day 84 . 
A second study was conducted to assess long-term PIZV immunogenicity and efficacy. Flavivirus seronegative male rhesus macaques $(n=4)$ were vaccinated with the $10 \mu \mathrm{g}$ PIZV dose on days 1 and 29 and challenged subcutaneously with $10^{4} \mathrm{ffu} / 0.5 \mathrm{~mL}$ ZIKV PRVABC59 on day 371 . Serum samples were collected and tested for antibody titers monthly up to 1 year post-vaccination and 30 days following ZIKV challenge (study day 401). To assess replication of Zika vRNA following ZIKV challenge, serum samples were collected on study days 371-381, and 385.

Challenge virus. Zika virus Puerto Rico strain PRVABC59 was used for the challenge $(0.5 \mathrm{~mL}$ containing a nominal dose of $10^{4} \mathrm{ffu}$ ). The seed stock was passaged three times on Vero cells at CDC, Fort Collins, Colorado. Virus stocks were prepared by passaging two times on C6/36 mosquito cells. Stocks were from a master working virus bank and tested for sterility, mycoplasma and endotoxin prior to use.

Animals. Indian rhesus macaques were screened for antibodies against flaviviruses, Herpes B Virus, Simian Retrovirus, Simian Immunodeficiency Virus, Simian T Lymphotropic Virus, Mycobacterium Tuberculosis, Simian Varicella Virus, Malaria, Salmonella, Shigella, Yersinia and internal parasites. Macaques were housed at either Charles River Laboratories or Inotiv. All in-life practices were approved and conducted per the Institutional Animal Care and Use Committee protocols (protocol number 2715-001 and 2715-002, or 2384-14376, respectively). Animals were evaluated twice daily for clinical signs following vaccination and challenge.

Screening. To select flavivirus naïve macaques, baseline IgG serostatus was determined using a multiplex Luminex kit (Ampersand Biosciences Flavivirus Serological Panel). Briefly, macaque serum was diluted 1:2,000 in sample diluent and added to multiplexed magnetic beads coupled with Zika, Dengue, Yellow Fever, Japanese Encephalitis, West Nile, Usutu, Saint Louis Encephalitis, and Chikungunya virus antigens. Serum and beads were incubated on a plate shaker for 1 hour at room temperature and then washed three times with assay buffer. Detection antibody, anti-IgG PE, was added to each sample and incubated on a plate shaker for 30 minutes at room temperature. Following another three wash cycles, beads were resuspended in assay buffer and read on the Bio-Plex MAGPIX to measure median fluorescent intensity (MFI) for each bead set. Macaques were considered flavivirus naïve and selected for the study if the MFI was below pre-defined assay cutoff criteria for seronegativity for all antigens.

Neutralizing antibodies. A Zika RVP assay (Sonnberg et al., manuscript in preparation) was used to determine neutralizing antibody titers in serum following the administration of PIZV (study days 1, 29, 57, and 71), and 30 days post-ZIKV challenge (day 101). Briefly, macaque serum was heat inactivated at $56{ }^{\circ} \mathrm{C}$ for 30 minutes and then serially diluted 3-fold in assay media for an 11-point dilution series. Diluted serum and RVP were plated in duplicate in a 384-well assay plate and incubated for 1 hour at $37^{\circ} \mathrm{C}$. Vero cells were added to each well and incubated at $37^{\circ} \mathrm{C}$ for 72 hours. Renilla-Glo substrate (Promega, WI, USA) was then added to the plate and incubated for 15 minutes at room temperature. Finally, plates were analyzed by a luminometer. The effective concentration at 50\% (EC50), was determined by a non-linear regression curve fit with the lower asymptote constrained to 0 in GraphPad Prism. The LLOQ for the NHP assay is $2.12 \log _{10}$ EC50, below which the serum matrix interfered with the measurement. The upper limit of quantitation (ULOQ) of the standard assay is $5.0 \log _{10}$ EC50. Any samples returning titers $>$ ULOQ were retested with a higher initial pre-dilution.

Anti-Zika IgG binding antibodies. Anti-Zika IgG antibody levels were measured for study days 1, 29, 57, and 71 (prior to ZIKV challenge) using a Luminex based anti-Zika IgG assay. Heat-inactivated serum samples were diluted 1:100 in assay buffer and then serially diluted 4-fold for an 8-point dilution curve. Magnetic beads covalently coupled with PIZV antigen were added to each sample dilution in a 96-well plate and each sample dilution was tested in duplicate. Plates were incubated on a plate shaker for 90 minutes at room temperature and then washed two times with assay buffer. Diluted anti-Ig-PE detection antibody was then added to each sample and incubated on a plate shaker for 1 hour at room temperature. After two wash cycles, beads were resuspended in assay buffer and read on the Luminex FLEXMAP 3D to measure the MFI. The IgG antibody concentration was quantified using a reference standard serum with an assigned IgG concentration in units $/ \mathrm{mL}(\mathrm{U} / \mathrm{mL})$. The LLOQ for the assay is $1.5 \log _{10} \mathrm{U} / \mathrm{mL}$.

RT-qPCR. Total RNA from serum samples was extracted using the Qiagen BioRobot Universal Instrument and QIAamp Virus BioRobot MDx Kit (Qiagen; Valencia, CA). Extracted and purified RNA was evaluated in two separate RT-PCR assays. Primarily, Zika vRNA was detected and quantified using RT-qPCR with primers and probe specific to known ZIKV genotypes as described in Lanciotti et al. ${ }^{33}$ and a standard curve generated from synthetic reference Zika vRNA (ATCC). A qualitative extraction control RT-PCR was also performed. The extraction control RT-PCR utilized a primer/probe set, specific to Macaca mulatta C1GALT1C1L mRNA, confirmed adequate nucleic acid extraction from serum samples independent of Zika vRNA detection. The geometric mean, range of peak Zika vRNA detection, and the respective percentage of protected macaques (as defined by absence of vRNA) was calculated using quantities greater than the assay lower limit of quantitation of $2.9 \log _{10} \operatorname{copies} / \mathrm{mL}$. Samples with vRNA concentration lower than the assay limit of detection of $2.3 \log _{10} \operatorname{copies} / \mathrm{mL}$ were considered negative.

Correlate of protection. The mean neutralizing antibody titers $\left(\log _{10}\right.$ EC50) determined by Zika RVP assay was computed for each dose group at day 71 (day of ZIKV challenge). Zika vRNA copies/mL determined by RT-qPCR assay were computed for each dose and timepoint post-challenge (study days 71-81 and 84). Peak vRNA for each macaque was defined as the highest observed vRNA concentration across all timepoints tested. Macaques were considered protected if vRNA was not detected or was below the assay LLOQ for all timepoints 
tested. The correlate of protection was defined as the maximum neutralizing antibody titer across all unprotected macaques in this study. This definition was conservative in that some protected macaques could have neutralizing antibody titer levels below the correlate of protection due to overlap between the distributions of protected and unprotected macaques. Since the correlate of protection was not a statistical estimate, no confidence intervals were reported.

Statistical analysis. Statistical tests were performed using the R software version 3.5.1.; $\log _{10}$ neutralization titers were compared for selected pairs of days for each dose group using a paired two-sided t-test. At day 57, Tukey's test was used to compare all dose groups with each other. A two-sided Spearman's test was applied to the unprotected macaques to check for an association between Day 71 neutralizing antibodies titers and peak vRNA. Comparisons between selected pairs of days were also performed with the $\log _{10} \operatorname{IgG}$ antibody levels in the main study and with the $\log _{10}$ neutralization titers from the long-term immunogenicity study using paired two-sided t-tests. A p-value threshold of 0.05 was used to determine statistical significance.

\section{Data availability}

The data that support the findings of this study are available from the corresponding author upon reasonable request and with permission of Takeda Vaccines, Inc.

Received: 17 December 2019; Accepted: 12 February 2020;

Published online: 26 February 2020

\section{References}

1. Rice, M. E. et al. Vital Signs: Zika-Associated Birth Defects and Neurodevelopmental Abnormalities Possibly Associated with Congenital Zika Virus Infection - U.S. Territories and Freely Associated States, 2018. MMWR Morb. Mortal. Wkly. Rep. 67, 858-867, https://doi.org/10.15585/mmwr.mm6731e1 (2018).

2. WHO Region of the Americas/Pan American Health Organization. PLISA Health Information Platform for the Americas: Cases of Zika virus disease, by country or territory, http://www.paho.org/data/index.php/en/mnu-topics/zika/524-zika-weekly-en.html (Accessed 27Aug2019).

3. Gruber, M. F. et al. Clinical Development Strategies and Considerations for Zika Vaccine Licensure. J. Infect. Dis. 216, S964-S970, https://doi.org/10.1093/infdis/jix433 (2017).

4. Plotkin, S. A. Correlates of protection induced by vaccination. Clin. Vaccine Immunol. 17, 1055-1065, https://doi.org/10.1128/ CVI.00131-10 (2010).

5. Vannice, K. S. et al. Demonstrating vaccine effectiveness during a waning epidemic: A WHO/NIH meeting report on approaches to development and licensure of Zika vaccine candidates. Vaccine 37, 863-868, https://doi.org/10.1016/j.vaccine.2018.12.040 (2019).

6. Abbink, P. et al. Protective efficacy of multiple vaccine platforms against Zika virus challenge in rhesus monkeys. Sci. 353, 1129-1132, https://doi.org/10.1126/science.aah6157 (2016).

7. Dowd, K. A. et al. Rapid development of a DNA vaccine for Zika virus. Sci. 354, 237-240, https://doi.org/10.1126/science.aai9137 (2016).

8. Richner, J. M. et al. Modified mRNA Vaccines Protect against Zika Virus Infection. Cell 169, 176, https://doi.org/10.1016/j. cell.2017.03.016 (2017).

9. Rayner, J. O. et al. Comparative Pathogenesis of Asian and African-Lineage Zika Virus in Indian Rhesus Macaque’s and Development of a Non-Human Primate Model Suitable for the Evaluation of New Drugs and Vaccines. Viruses 10, https://doi.org/10.3390/ v10050229 (2018).

10. Osuna, C. E. \& Whitney, J. B. Nonhuman Primate Models of Zika Virus Infection, Immunity, and Therapeutic Development. J. Infect. Dis. 216, S928-S934, https://doi.org/10.1093/infdis/jix540 (2017).

11. Newman, C., Friedrich, T. C. \& O’Connor, D. H. Macaque monkeys in Zika virus research: 1947-present. Curr. Opin. Virol. 25, 34-40, https://doi.org/10.1016/j.coviro.2017.06.011 (2017).

12. Li, X. F. et al. Characterization of a 2016 Clinical Isolate of Zika Virus in Non-human Primates. EBioMedicine 12, 170-177, https:// doi.org/10.1016/j.ebiom.2016.09.022 (2016).

13. Aliota, M. T. et al. Heterologous Protection against Asian Zika Virus Challenge in Rhesus Macaques. PLoS Negl. Trop. Dis. 10, e0005168, https://doi.org/10.1371/journal.pntd.0005168 (2016).

14. Dudley, D. M. et al. A rhesus macaque model of Asian-lineage Zika virus infection. Nat. Commun. 7, 12204, https://doi.org/10.1038/ ncomms12204 (2016).

15. Hirsch, A. J. et al. Zika Virus infection of rhesus macaques leads to viral persistence in multiple tissues. PLoS Pathog. 13, e1006219, https://doi.org/10.1371/journal.ppat.1006219 (2017).

16. Baldwin, W. R. et al. Purified Inactivated Zika Vaccine Candidates Afford Protection against Lethal Challenge in Mice. Sci. Rep. 8, 16509, https://doi.org/10.1038/s41598-018-34735-7 (2018).

17. Sumathy, K. et al. Protective efficacy of Zika vaccine in AG129 mouse model. Sci. Rep. 7, 46375, https://doi.org/10.1038/srep46375 (2017).

18. Pardi, N. et al. Zika virus protection by a single low-dose nucleoside-modified mRNA vaccination. Nat. 543, 248-251, https://doi. org/10.1038/nature21428 (2017).

19. Muthumani, K. et al. In vivo protection against ZIKV infection and pathogenesis through passive antibody transfer and active immunisation with a prMEnv DNA vaccine. NPJ Vaccines 1, 16021, https://doi.org/10.1038/npjvaccines.2016.21 (2016).

20. Larocca, R. A. et al. Vaccine protection against Zika virus from Brazil. Nat. 536, 474-478, https://doi.org/10.1038/nature 18952 (2016).

21. Abbink, P. et al. Durability and correlates of vaccine protection against Zika virus in rhesus monkeys. Sci Transl Med $\mathbf{9}$, https://doi. org/10.1126/scitranslmed.aao4163 (2017).

22. Espinosa, D. et al. Passive Transfer of Immune Sera Induced by a Zika Virus-Like Particle Vaccine Protects AG129 Mice Against Lethal Zika Virus Challenge. EBioMedicine 27, 61-70, https://doi.org/10.1016/j.ebiom.2017.12.010 (2018).

23. Hombach, J., Solomon, T., Kurane, I., Jacobson, J. \& Wood, D. Report on a WHO consultation on immunological endpoints for evaluation of new Japanese encephalitis vaccines, WHO, Geneva, 2-3 September, 2004. Vaccine 23, 5205-5211, https://doi. org/10.1016/j.vaccine.2005.07.002 (2005).

24. Mason, R. A., Tauraso, N. M., Spertzel, R. O. \& Ginn, R. K. Yellow fever vaccine: direct challenge of monkeys given graded doses of $17 \mathrm{D}$ vaccine. Appl. Microbiol. 25, 539-544 (1973).

25. Kreil, T. R., Maier, E., Fraiss, S. \& Eibl, M. M. Neutralizing antibodies protect against lethal flavivirus challenge but allow for the development of active humoral immunity to a nonstructural virus protein. J. Virol. 72, 3076-3081 (1998). 
26. Modjarrad, K. et al. Preliminary aggregate safety and immunogenicity results from three trials of a purified inactivated Zika virus vaccine candidate: phase 1, randomised, double-blind, placebo-controlled clinical trials. Lancet 391, 563-571, https://doi. org/10.1016/S0140-6736(17)33106-9 (2018).

27. Nazerai, L., Pravsgaard Christensen, J. \& Randrup Thomsen, A. A 'Furry-Tale' of Zika Virus Infection: What Have We Learned from Animal Models? Viruses 11, https://doi.org/10.3390/v11010029 (2019).

28. Lanciotti, R. S., Lambert, A. J., Holodniy, M., Saavedra, S. \& Signor Ldel, C. Phylogeny of Zika Virus in Western Hemisphere, 2015. Emerg. Infect. Dis. 22, 933-935, https://doi.org/10.3201/eid2205.160065 (2016).

29. Dowd, K. A. et al. Broadly Neutralizing Activity of Zika Virus-Immune Sera Identifies a Single Viral Serotype. Cell Rep. 16, 1485-1491, https://doi.org/10.1016/j.celrep.2016.07.049 (2016)

30. Sariol, C. A., Nogueira, M. L. \& Vasilakis, N. A Tale of Two Viruses: Does Heterologous Flavivirus Immunity Enhance Zika Disease? Trends Microbiol. 26, 186-190, https://doi.org/10.1016/j.tim.2017.10.004 (2018)

31. Osuna, C. E. et al. Zika viral dynamics and shedding in rhesus and cynomolgus macaques. Nat. Med. 22, 1448-1455, https://doi. org/10.1038/nm.4206 (2016).

32. Golding, H., Khurana, S. \& Zaitseva, M. What Is the Predictive Value of Animal Models for Vaccine Efficacy in Humans? The Importance of Bridging Studies and Species-Independent Correlates of Protection. Cold Spring Harb Perspect Biol 10, https://doi. org/10.1101/cshperspect.a028902 (2018).

33. Lanciotti, R. S. et al. Genetic and serologic properties of Zika virus associated with an epidemic, Yap State, Micronesia, 2007. Emerg. Infect. Dis. 14, 1232-1239, https://doi.org/10.3201/eid1408.080287 (2008).

\section{Acknowledgements}

We would like to acknowledge Michael Johnson, Jill Livengood, Lovkesh Karwal and Raman Rao for vaccine and challenge virus preparation, Lydia Anderson, Amanda Brinkman, Hui-Ling Chen, Dan Shepherd, Katelyn Bartlett, Joseph Lee and Tim Powell for assay support, and Boris Predovich, Todd Haryu, Stephene Rose, James Justen, Jennifer Ruger, and Tyler Plachta for in vivo study support. This project was funded with Federal funds from the Department of Health and Human Services; Office of the Assistant Secretary for Preparedness and Response; Biomedical Advanced Research and Development Authority, under Contract No. HHSO100201600015C and by Takeda Vaccines, Inc.

\section{Author contributions}

G.Y., H.K.P. and H.J.D. designed the studies. G.Y., K.J.B., M.Z., K.M., S. Sanisetty and S. Sonnberg performed assays and analyzed data. G.H. analyzed data and performed statistical analyses. G.Y., S.T. and C.S.G. managed in vivo work. H.K.P. and H.J.D. provided program oversight. G.Y. and H.J.D. wrote the paper with all co-authors. All authors approved the final manuscript.

\section{Competing interests}

G.Y., K.J.B., M.Z., G.H., S. Sonnberg, H.K.P. and H.J.D. are employees of Takeda. S. Sanisetty was a contract researcher on assignment with Takeda. S.T. works as an employee of Charles River Laboratories, contracted by Takeda. K.M. works as an employee of Viracor Eurofins, contracted by Takeda. C.S.G. works as an employee of Inotiv, contracted by Takeda.

\section{Additional information}

Correspondence and requests for materials should be addressed to G.Y.

Reprints and permissions information is available at www.nature.com/reprints.

Publisher's note Springer Nature remains neutral with regard to jurisdictional claims in published maps and institutional affiliations.

(c) (i) Open Access This article is licensed under a Creative Commons Attribution 4.0 International License, which permits use, sharing, adaptation, distribution and reproduction in any medium or format, as long as you give appropriate credit to the original author(s) and the source, provide a link to the Creative Commons license, and indicate if changes were made. The images or other third party material in this article are included in the article's Creative Commons license, unless indicated otherwise in a credit line to the material. If material is not included in the article's Creative Commons license and your intended use is not permitted by statutory regulation or exceeds the permitted use, you will need to obtain permission directly from the copyright holder. To view a copy of this license, visit http://creativecommons.org/licenses/by/4.0/.

(c) The Author(s) 2020 